\title{
III-V Photonic Crystal Wire Cavity Laser on Silicon wafer
}

\author{
Yacine Halioua, ${ }^{1,2, *}$ Alexandre Bazin, ${ }^{1}$ Paul Monnier, ${ }^{1}$ Timothy J. Karle, ${ }^{1}$ Isabelle Sagnes, ${ }^{1}$ \\ Gunther Roelkens, ${ }^{2}$ Dries Van Thourhout, ${ }^{2}$ Fabrice Raineri, ${ }^{1,3}$ Rama Raj $^{1}$ \\ ${ }^{1}$ Laboratoire de Photonique et de Nanostructures, CNRS-UPR20, Route de Nozay, 91460 \\ Marcoussis, France \\ ${ }_{2}^{2}$ Photonics Research Group, Department of Information Technology, Ghent University \\ B-9000 Ghent, Belgium \\ ${ }^{3}$ Université Paris Denis Diderot, 75205 Paris, France \\ *Corresponding author: yacine.halioua@lpn.cnrs.fr
}

We report on the modelling study and experimental characterization of an InP-based photonic crystal wire cavity laser bonded to silicon. Calculated Q-factors are of the order of 104.

Simulation gives an insight into the variation of the resonant mode displacement against the length of the cavity and the dependence of the Q-factor on the geometrical parameters of the laser cavity. Low threshold laser operation is demonstrated.

OCIS codes: (350.4238) Nanophotonics and photonic crystals, (140.3460) Lasers, (130.3120) Integrated optics devices 


\section{INTRODUCTION}

Heterogeneous integration of III-V semiconductor devices on silicon has gained considerable momentum in the last few years due to the drive towards obtaining laser sources in a CMOS compatible platform $[1,2]$. The introduction of nanolasers, such as photonic crystal III-V semiconductor lasers $(\mathrm{PhC})$ [3], would enable high speed operation $[4,5]$ and reduced power consumption [6] with considerable reduction in footprint. The integration of photonic crystal lasers onto silicon has recently been demonstrated using different wafer bonding techniques such as $\mathrm{SiO}_{2}-\mathrm{SiO}_{2}$ molecular bonding [7], Au-In eutectic bonding [8] or adhesive bonding [9]. In all these structures, the PhC slab is separated from the Si substrate by a low-refractive index (low-n) layer $\left(\mathrm{SiO}_{2}\right.$ or $\left.\mathrm{BCB}\right)$ for the vertical confinement of light. The presence of this layer reduces the optical confinement (compared to an air-clad membrane) making it less straightforward to obtain high Q cavities, such as those demonstrated in suspended membranes [10]. This is one of the reasons why previously demonstrated lasers rely principally upon low-group velocity modes either at a band edge $[7,8]$ or at a waveguide mode edge $[11,12]$.

In this work, we report on the simulation and fabrication (see fig. 1) of active InP-based photonic crystal wire nanocavity lasers bonded to SOI. These small volume cavities made of a single row of holes drilled in a waveguide [13] enable indeed record high Q/V ratios, even in the case where the substrate is a low-n layer such as silica. So far these structures were made in passive materials such as $\mathrm{Si}[14,15]$. These cavities should also be excellent candidates for enhancing nonlinear effects including gain. So in order to explore the possibilities that these cavities offer for laser emission, we study both numerically and experimentally their performance by varying their geometrical parameters. Particular attention is given to the length of the cavity as it determines the resonance wavelength and the quality factor. The close 
matching between the expected and observed results attest to the fine tuned simulation and the accuracy of the fabrication both of which lead finally to the demonstration of efficient low threshold laser operation.

\section{DESIGN AND MODELLING OF THE WIRE CAVITIES}

The cavities under investigation are made in a $0.55 \mu \mathrm{m}$-wide $0.26 \mu \mathrm{m}$-thick InP strip waveguide incorporating four InGaAs/InGaAsP quantum wells (QWs) as an active medium. Two sets of holes are drilled along the waveguide, spaced by a gap of length $L$ to form a cavity. The length $L$ is varied to obtain different cavity lengths. Each high reflectivity mirror comprises 11 holes, a periodic section of 8 holes and at the edge of the cavity defect zone, the remaining three holes are made with gradually decreasing diameters to form a taper. Laterally, light is confined within the cavity by index guiding due to the semiconductor/air interface, and in the vertical direction by air above and BCB below. Our wire cavity structure is bonded to an unpatterned silicon wafer by a transparent $1 \mu \mathrm{m}$ thick low index $\mathrm{BCB}$ joint layer $(\mathrm{n}=1.54$ at $1.55 \mu \mathrm{m})$. The schematics are given in Fig. 1.

The resonance wavelength is determined by several parameters: the length of the cavity, the effective index of the mode confined in the wire and the phase change induced by the mirror. In these kinds of structures it has been shown that high $Q$ values may be obtained by tapering the size of the first 3 holes on either side of the cavity [16]. The tapering is designed to adiabatically minimise the modal mismatch between the propagating guided mode and the evanescent mirror modes, thereby decreasing the losses at the cavity/mirror interface and thus increasing the reflectivity.

We optimize our structure using 3D FDTD [17] using, as a starting point, a cavity design which has already been demonstrated to possess a high Q in the SOI material system [14]. The 
design is pertinently modified in order to incorporate all the relevant material and geometrical parameters of our desired InP-based structure. By iterating the radius and period which give the largest band gap and therefore the best confinement we designed the wire cavity lattices in the InP-based system. We then move the spectral position of the center of the high reflectivity mirror band to $1.55 \mu \mathrm{m}$ where maximum gain is available in our active layer. From the simulation for the mirror zone, we get $97 \mathrm{~nm}$ radius holes with a pitch of $376 \mathrm{~nm}$, and for the tapered zone we get radius $=69 \mathrm{~nm}$, period $=320 \mathrm{~nm}$ for the first hole, radius $=85.5 \mathrm{~nm}$, period $=337 \mathrm{~nm}$ for the second hole and radius $=99 \mathrm{~nm}$, period $=376.5 \mathrm{~nm}$ for the third one. We then calculate, as a function of the cavity length, the 4 essential parameters: the resonant wavelengths, the $\mathrm{Q}$ factor, the modal volume and the confinement factor.

The resonant wavelengths are plotted in Fig. 2. Two orders of the Fabry-Perot modes for the symmetric (triangles) and anti-symmetric (squares) modes are represented (see symmetry axis shown on Fig. 1). The dispersion ( $\mathrm{d} \lambda / \mathrm{dL}$, where $\mathrm{L}$ is the length of the cavity) is mostly due to the wavelength dependent variation of the penetration depth into the mirrors as confirmed from 2D simulations (not shown here). Typical Fabry Perot resonator behaviour is observed below $1555 \mathrm{~nm}$, with the resonance wavelength of the cavity shifting to higher values linearly with increasing cavity length. However, the curves show an inflection close to the band-edge of the mirrors situated around $1555 \mathrm{~nm}$ (valence band-edge), beyond which varying the length has little effect on the wavelength: the field is not tightly confined into the defect any longer and leaks through the mirrors. The dispersion is now close to the one of the valence band edge mode. Further, no FP modes are seen for wavelength less than $1360 \mathrm{~nm}$ as they fall outside the $\mathrm{PhC}$ band gap. 
In Fig. 3a we plot in triangles the simulated Q factor versus the cavity length corresponding to the symmetric mode indicated by the arrow in Fig. 2. It is seen, for the second order symmetric mode, that the $\mathrm{Q}$ attains in our system a maximum of $1.4 \times 10^{4}$ for a cavity length of $1 \mu \mathrm{m}$ giving a resonance at $1568 \mathrm{~nm}$. We expect the maximum $\mathrm{Q}$ factor is reached when the mirror reflectivity is maximum. As indicated in [16], this is found at wavelengths where the best modal matching between the cavity and the mirror is obtained thanks to the tapered zone, fixing thereby the cavity lengths which give the best Q factor. The Q-curve shows a smooth rise for lower wavelengths whereas it falls off rather abruptly for longer wavelengths as the wavelength associated with the highest Q cavity mode is closer to the valence band edge of the mirrors as can be seen in Fig. 2. We also calculate the modal volume [18] of the cavities using 3D FDTD and plot it as a function of the resonant wavelength on Fig. 3a) (dots) and as function of the cavity length on Fig. 3b) (dots). We obtain volumes ranging from 0.38 to $0.92(\lambda / n)^{3}$ for cavities lengths of $0.7 \mu \mathrm{m}(\lambda=1351 \mathrm{~nm})$ to $1.3 \mu \mathrm{m}(\lambda=1636 \mathrm{~nm})$, quantities close to the limit of a cubic halfwavelength in the material $(\lambda / 2 n)^{3}$. The volume increases rapidly with the length and the wavelength up to $\mathrm{L}=1.05 \mu \mathrm{m}$ and is then almost constant for $\mathrm{L}>1.05 \mu \mathrm{m}$. Finally, we calculate the confinement factor $\Gamma$ which is defined as the ratio of the field intensity confined in the semiconductor active material (here 4 QWs) to the total intensity stored in the mode. This parameter is important when laser emission is considered as only the field overlapped with the active material is directly amplified. As seen in Fig. $3 b$ ) (squares), $\Gamma$ varies from 0.243 to 0.252 , meaning that only a variation of $3 \%$ is obtained for the whole set of cavities. 


\section{FABRICATION AND OPTICAL CHARACTERIZATION}

The fabricated structure consists of a $260 \mathrm{~nm}$-thick InP membrane bonded on an unpatterned silicon wafer using a $1 \mu \mathrm{m}$ thick layer of $\mathrm{BCB}(\mathrm{n}=1.54)$ [19]. The InP heterostucture with 4 quantum wells grown by MOCVD exhibits photoluminescence (PL) which peaks around 1.55 $\mu \mathrm{m}$ (see inset of Fig. 2). The wire cavity is obtained using electron beam lithography followed by plasma etching. Details of the fabrication may be found in [20]. As can be seen on the SEM image in Fig. 1, the wire cavity is isolated from the rest of the InP slab by a $1 \mu \mathrm{m}$-wide air trench made around it for lateral confinement. The cavity size is varied between $465 \mathrm{~nm}$ and $1465 \mathrm{~nm}$ in steps of $25 \mathrm{~nm}$ in order to obtain resonances over the full range of the gain.

We explore the fabricated samples at room temperature by measuring the light emitted under optical pumping. The samples are surface pumped using a 50x IR long working distance objective which focus the pump light down to a spot size around $1.5 \mu \mathrm{m}$. The laser source for the pump is a diode laser at $808 \mathrm{~nm}$ modulated to produce $10 \mathrm{~ns}$ pulses at $200 \mathrm{kHz}$ repetition rate. These characteristics are chosen so as to reduce device heating. The NIR pump is absorbed by each of the semiconductor materials, InP, InGaAs QWs and their InGaAsP barriers. The light emission is collected by the very same objective, separated from the pump by a dichroic mirror and is analyzed using a spectrometer equipped with a cooled InGaAs photodiodes array.

For each cavity, the wavelength of the peaks observed in the PL spectra is plotted on Fig 2 with dots as a function of the cavity length. Resonances are observed over the entire QWs gain bandwidth, i.e. from $1373 \mathrm{~nm}$ to $1575 \mathrm{~nm}$. The experimental measurements agree very well with the simulation predictions. We see that for cavities with lengths between 0.78 to $1.05 \mu \mathrm{m}$ the experimental results closely follow the $\mathrm{d} \lambda / d L$ trend from simulation. For longer cavities, the resonant wavelengths follow also the simulated cavity mode dispersion closely. But, for very 
short cavities, larger $\mathrm{d} \lambda / \mathrm{dL}$ are obtained because the emission wavelength is more susceptible to phase and amplitude perturbations arising from changes in the waveguide width and the size and position of the holes immediately surrounding the cavity.

Laser operation is obtained over a wide range of wavelengths with a threshold ranging from $0.86 \mathrm{~mW}$ to $8.8 \mathrm{~mW}$ peak power (total input power on the sample). As an example of laser operation, we plot on Fig. 5 the variation of the emitted power (squares) as a function of the pump peak power on log-log scale for one cavity (cavity length $=960 \mathrm{~nm}, 1520 \mathrm{~nm}$ lasing wavelength). We observe the typical s-shape attesting to the transition from spontaneous emission to stimulated emission [21] here at $1.2 \mathrm{~mW}$. As seen in Fig 5, the FWHM of the emission (dots) decreases as the pump power is increased towards the threshold, attaining a minimum value of $0.26 \mathrm{~nm}$. Then, as the pump power is further increased the linewidth increases. These measurements are performed for each of the cavities in order to deduce the corresponding threshold peak pump powers. On Fig. 4 we plot the measured peak pump powers at threshold as a function of the laser wavelength, the measurements correspond to the second order longitudinally symmetric mode, the third sets of experimental points on Fig. 2. The laser threshold diminishes by a factor of around 2.5 as the cavity emission shifts from $1437 \mathrm{~nm}$ (cavity length $=0.8 \mu \mathrm{m}$ ) up to $1515 \mathrm{~nm}$ for a cavity length of $0.94 \mu \mathrm{m}$ hitting a minimum threshold peak

power of $\quad 0.8 \mathrm{~mW}$. Then, as the cavity mode goes beyond $1539 \mathrm{~nm}$ (cavity length $>1 \mu \mathrm{m}$ ), the thresholds increase sharply.

\section{DISCUSSION AND CONCLUSIONS}

In order to establish a relationship between the Q factor and the optimal operation of these lasers, the numerically obtained $\mathrm{Q} / \mathrm{V}$ ratios are compared to laser thresholds. The threshold 
varies inversely with the cavity photon density [21]. To take into account the variation in operation wavelengths of the different cavities, Q/V is multiplied by the material gain $G(\lambda)$ which is derived here from the PL spectrum of the QWs, and by the inverse of the confinement factor. We plot V/(Q.G( $\lambda) \cdot \Gamma)$ as a function of the wavelength on Fig. 4. The shape of this curve shows a close fit with the behaviour of the laser threshold. As the general trend is preserved, the minimum value of $\mathrm{V} /(\mathrm{Q} \cdot \mathrm{G}(\lambda) \cdot \Gamma)$ is obtained at $1538 \mathrm{~nm}, 15 \mathrm{~nm}$ away from the wavelength corresponding to the minimum threshold. The two curves separate for $\lambda>1500 \mathrm{~nm}$ which may be explained by the discrepancy observed between the simulation and the experiment in the value of the resonant wavelength as seen in Fig.2.

In conclusion, we have demonstrated lasing operation with fairly low threshold of InPbased wire cavity structures on a silicon substrate from wavelength from $1437 \mathrm{~nm}$ to $1565 \mathrm{~nm}$. The influence of the cavity length over the Q factor, the resonant wavelength, the modal volume and the confinement factor was meticulously analysed both numerically and experimentally. Our results show that an optimal value for the cavity length can be found to obtain the lowest laser threshold. Moreover, the close correspondence between the experimental measurements and the numerical calculations illustrates the quality and the accuracy of the technological processing.

The results are a clear demonstration that III-V wire cavities are excellent candidates for ultra compact ( $\mu \mathrm{m}^{2}$ scale footprint) building blocks in active nanophotonics on silicon as it is possible to obtain high $\mathrm{Q}$ factors in such a substrate based hybrid system possessing both mechanical and thermal stability.

\section{ACKNOWLEDGEMENTS:}


We thank R. Braive for the etching of the photonic crystals and F. Bordas for useful discussions. This work was partly funded by FP7 ICT European project HISTORIC and ANR jeunes chercheurs PROWOC.

\section{References:}

[1] A. W. Fang, H. Park, Y. Kuo, R. Jones, O. Cohen, D. Liang, O. Raday, M. N. Sysak, M. J. Paniccia and J. E. Bowers,"Hybrid evanescent silicon Devices" Materials Today 10,28-35, (2007)

[2] J. Van Campenhout, P. Rojo Romeo, P. Regreny, C. Seassal, D. Van Thourouth, S. Verstuyft, L. Di Cioccio, J.-M. Fedeli, C. Lagahe and R. Baets, "Electrically pumped InP-based microdisk lasers integrated with a nanophotonic silicon-oninsulator waveguide circuit", Opt. Express 15, 6744 (2007)

[3] O. Painter, R. K. Lee, A. Yariv, A. Scherer, J. D. O’Brien, P.D. Dapkus, I. Kim, “Two-Dimensional Photonic Band-Gap Defect Mode Laser”, Science, 284 (5421), 1819-1821, Jun. 1999

[4] H. Altug, D. Englund and J. Vukovic, "Ultrafast photonic crystal nanocavity laser” Nature Physics 2, $484-488$ (2006)

[5] F. Raineri, A. Yacomotti, T. J. Karle, R. Hostein, R. Braive, A. Beveratos, I. Sagnes and R. Raj, "Dynamics of band-edge photonic crystal lasers", Optics Express 17, 3165 (2009) 
[6] K. Nozaki, S. Kita and T. Baba, "Room temperature continuous wave operation and controlled spontaneous emission in ultrasmall photonic crystal nanolaser" Optics express, vol. 15, no. 12, 2007

[7] C. Monat, C. Seassal, X. Letartre,P. Regreny, P. Rojo-Romeo, and P. Viktorovitch, "InP 2D photonic crystal microlasers on silicon wafer: room temperature operation at $1.55 \mu \mathrm{m} "$, Electron. Lett. 37, 764 (2001)

[ 8] G. Vecchi, F. Raineri, I. Sagnes, A. Yacomotti, P. Monnier, T. J. Karle, K.-H. Lee, R. Braive, L. Le Gratiet, S. Guilet, G. Beaudoin, A. Talneau, S. Bouchoule, J. A. Levenson and R. Raj, "Continuous-wave operation of photonic band-edge laser near $1.55 \mu \mathrm{m}$ on silicon wafer “, Optics Express, Vol. 15, Issue 12, pp. 7551$7556(2007)$

[9] G. Vecchi, F. Raineri, I. Sagnes, K.-H. Lee, S. Guilet, L. Le Gratiet, F. Van Laere, G. Roelkens, D. Van Thourhout, R. Bats, J. A. Levenson and R. Raj, "Photoniccrystal surface emitting laser near 1,55 micron on gold-coated silicon wafer “ Electron. Lett. 43, 343 (2007)

[10] Y. Tanaka, T. Asano, R. Hatsuna and S. Noda, "Investigation of point-defect cavity formed in two-dimensional photonic crystal slab with one-sided dielectric cladding”, Appl. Phys. Lett. 88, 011112 (2006)

[11] Y. Halioua, T. J. Karle, F. Raineri, P. Monnier, I. Sagnes, G. Roelkens, D. Van Thourhout, R. Raj, "Hybrid InP-based photonic crystal lasers on silicon on insulator wires”, Appl. Phys. Lett. 95, 201119 (2009)

[12] T.J. Karle, Y. Halioua, F. Raineri, P. Monnier, R. Braive, L. Le Gratiet, G. Beaudoin, I. Sagnes, G. Roelkens, F. Van Laere, D. Van Thourout and R. Raj, 
"Heterogeneous integration and precise alignement of InP-based photonic crystal lasers to complementary metal-oxide semiconductor fabricated silicon-oninsulator wire waveguides”, Journal of Applied Physics, 107 (6), p.8 (2010)

[13] J.S. Foresi, P. R. Villeneuve, J. Ferrera, E.R. Thoen, G. Steinmevert, S. Fan, J.D. Joannopoulos, L.C. Kimerling, H. I. Smith and E.P. Ippen, "Photonic-bandgap microcavities in optical waveguides", Nature 390 (1997)

[14] A. R. Zain, N. P. Johnson, M. Sorel and R. M. De La rue, "Ultra high quality factor one dimensional photonic crystal/photonic wire micro-cavities in siliconon-insulator (SOI)", Optics Express, vol. 16, no. 16 (2008)

[15] P. B. Deotare, M. W. McCutcheon, I. W. Frank, M. Khan, and M. Lončar, "High Quality factor photonic crystal nanobeam cavities”, Applied Physics Letters, 94, $121106(2009)$

[16] P. Velha, J.C. Rodier, P. Lalanne, J.P. Hugonin, E. Picard, T. Charvolin and E. Hadji, "Ultra-high-reflectivity photonic-bandgap mirrors in a ridge SOI waveguide", New Joural of Physics 8 (2006) 204

[17] We use FDTD Solutions by Lumerical http://www.lumerical.com

[18] J. T. Robinson, C. Manolatou, L. Chen and M. Lipson, "Ultrasmall Mode Volumes in Dielectric Optical Microcavities”, Phys. Rev. Lett. 95, 143901 (2005)

[19] G. Roelken, J. Brouckaert, D. Van Thourhout, R. Baets, R. Notzel and M. Smith, "Adhesive bonding of an $\mathrm{InP} / \mathrm{InGaAsP}$ dies to processed silicon-on-insulators wafers", Journal of the Electrochemical Society, 2006, 153 (12) 
[20] K.-H. Lee, S. Guilet, G. Patriarche, I. Sagnes, and A. Talneau, "Smooth sidewall in InP-based photonic crystal membrane etched by N2-based inductive coupled plasma”, J. Vac. Sci. Technol. B 26, 1326 (2008).

[21] E. Kapon, Semiconductor Lasers, Academic, New York, (1999). 


\section{FIGURE CAPTIONS:}

Figure 1

a) SEM image of the sample. The dotted line denotes the symmetry axis taken for the cavity mode - b) Schematics of the structure profile.

Figure 2:

Resonance wavelength against cavity length. The triangles and the square represent the results obtained by 3D FDTD simulation for the symmetric and the antisymmetric modes respectively. The dots are the experimental measurements.

Figure 3:

a) Q factor and modal volume extracted from the 3D FDTD simulations as a function of the resonance wavelength. The $\mathrm{Q}$ factor is plotted with triangles and the modal volume $\mathrm{V}$ with dots b) Modal volume and confinement factor versus cavity length. The modal volume $\mathrm{V}$ is plotted with dots and the modal volume with squares

Figure 4:

Measured threshold (dots) and calculated V/(Q.G $(\lambda) . \Gamma)$ (triangles) versus resonance wavelength Inset: Photoluminescence spectrum of the quantum wells

Figure 5:

Laser intensity (squares) and FWHM of the emitted light versus pump peak power. Inset: Spectrum of the laser emission. 
Figure 1

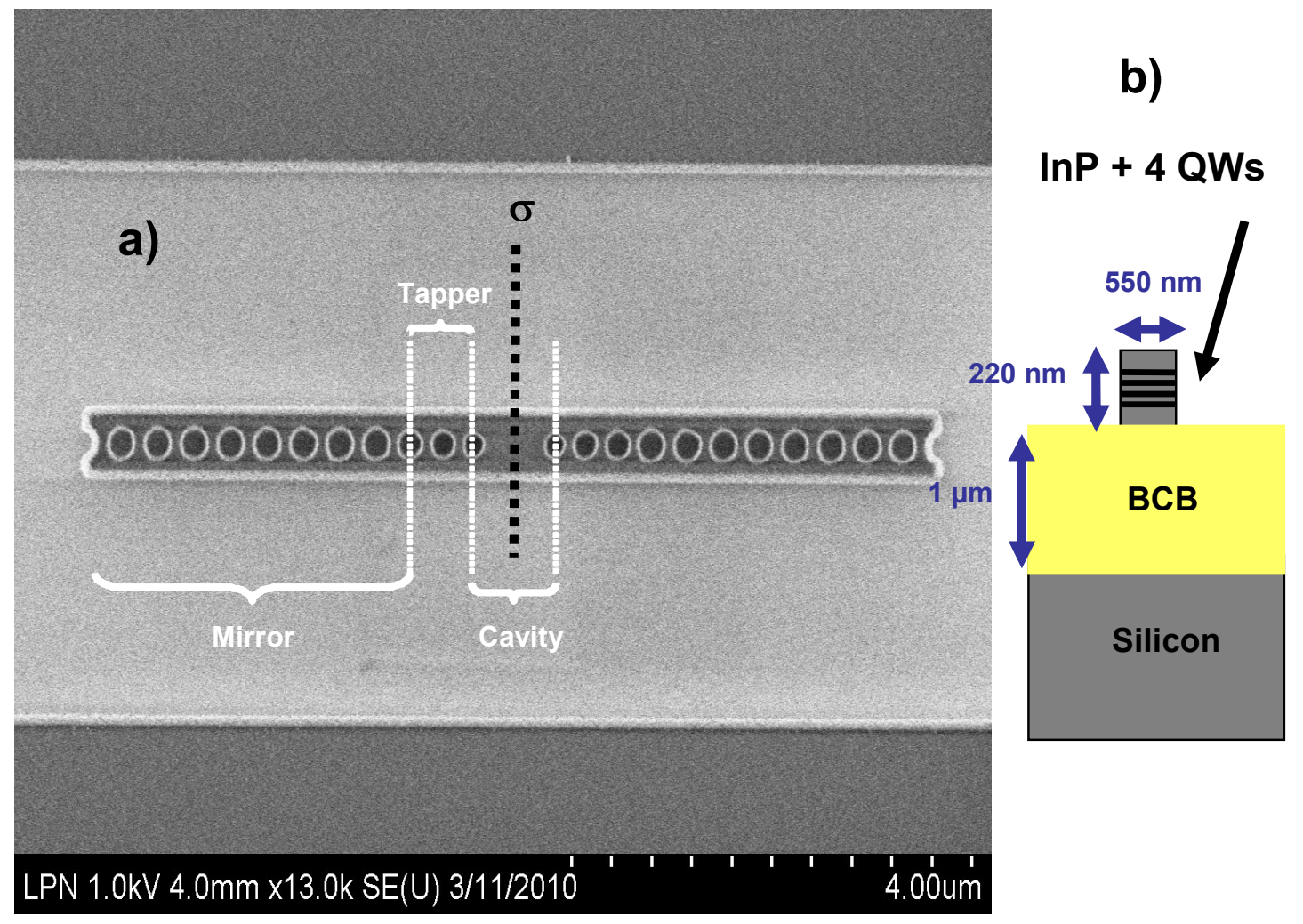


Figure 2

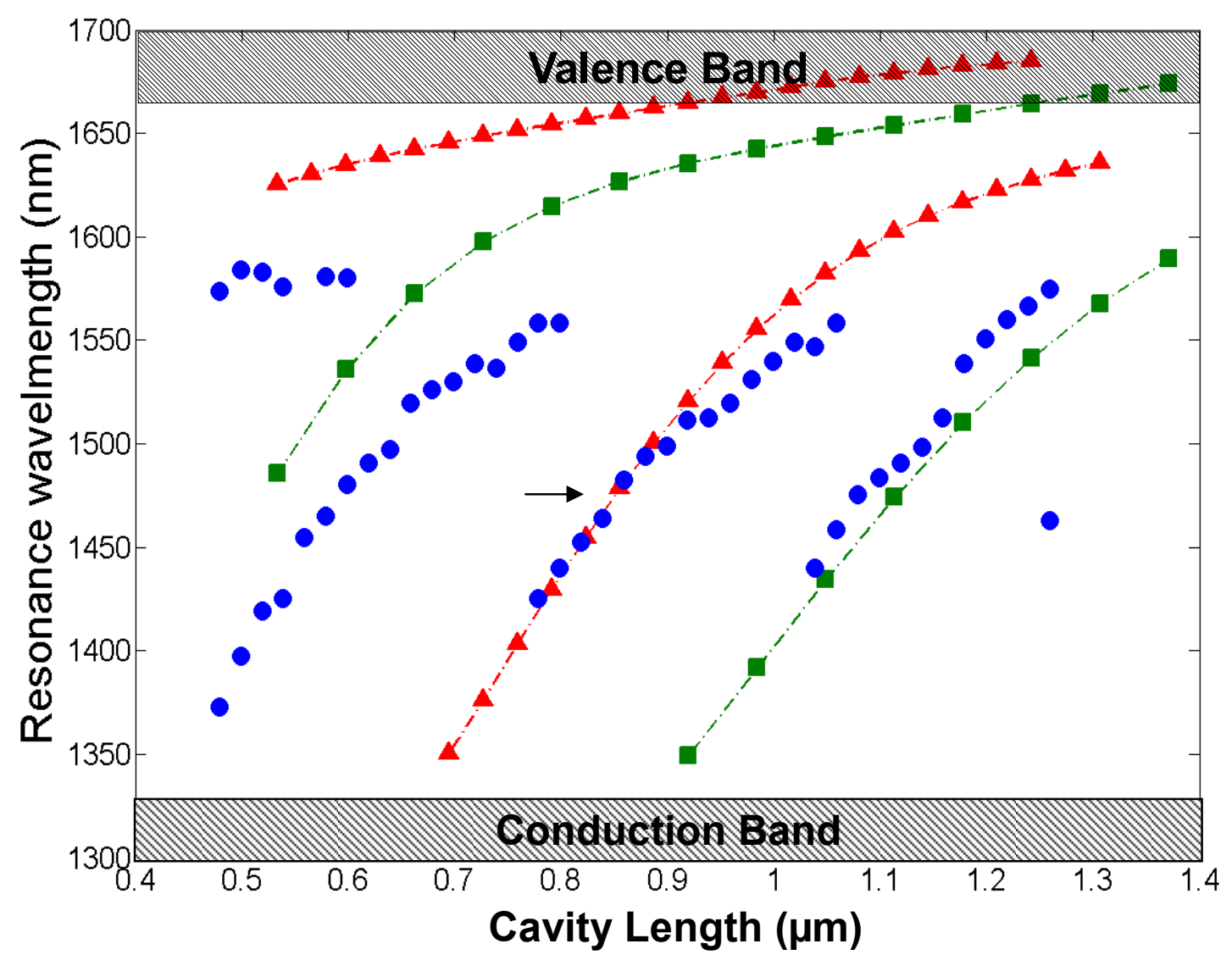


Figure 3

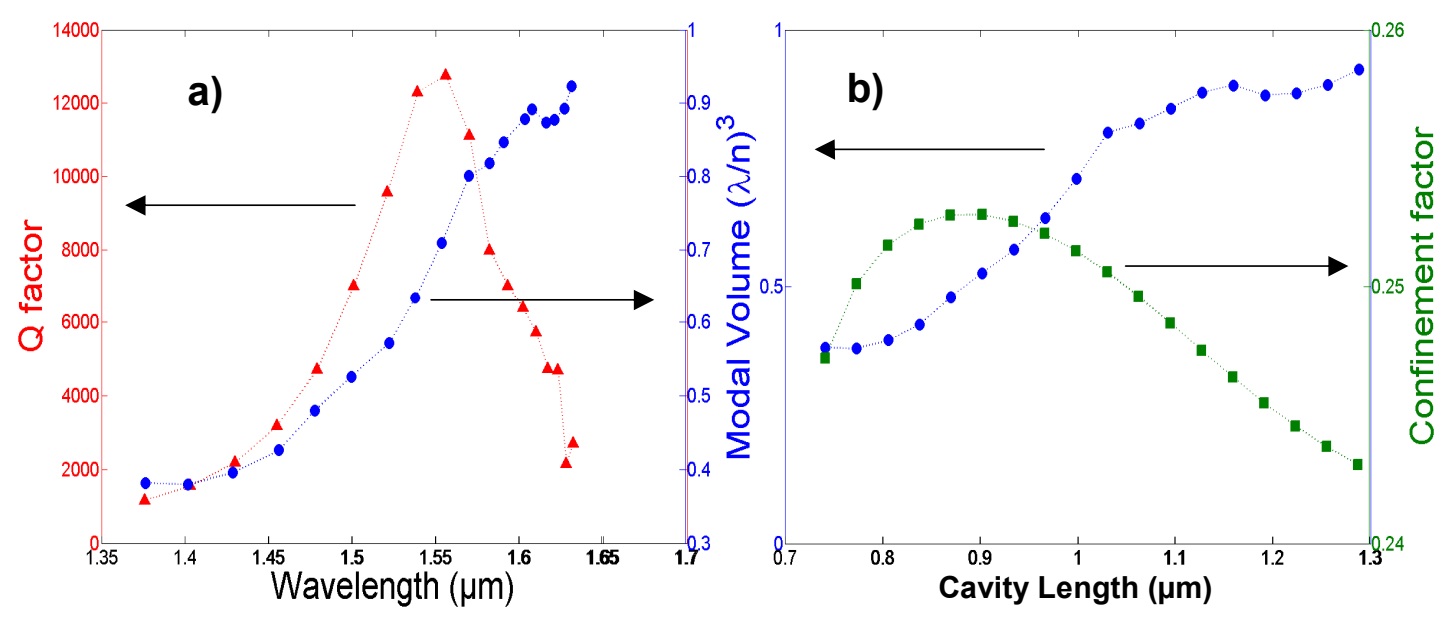


Figure 4

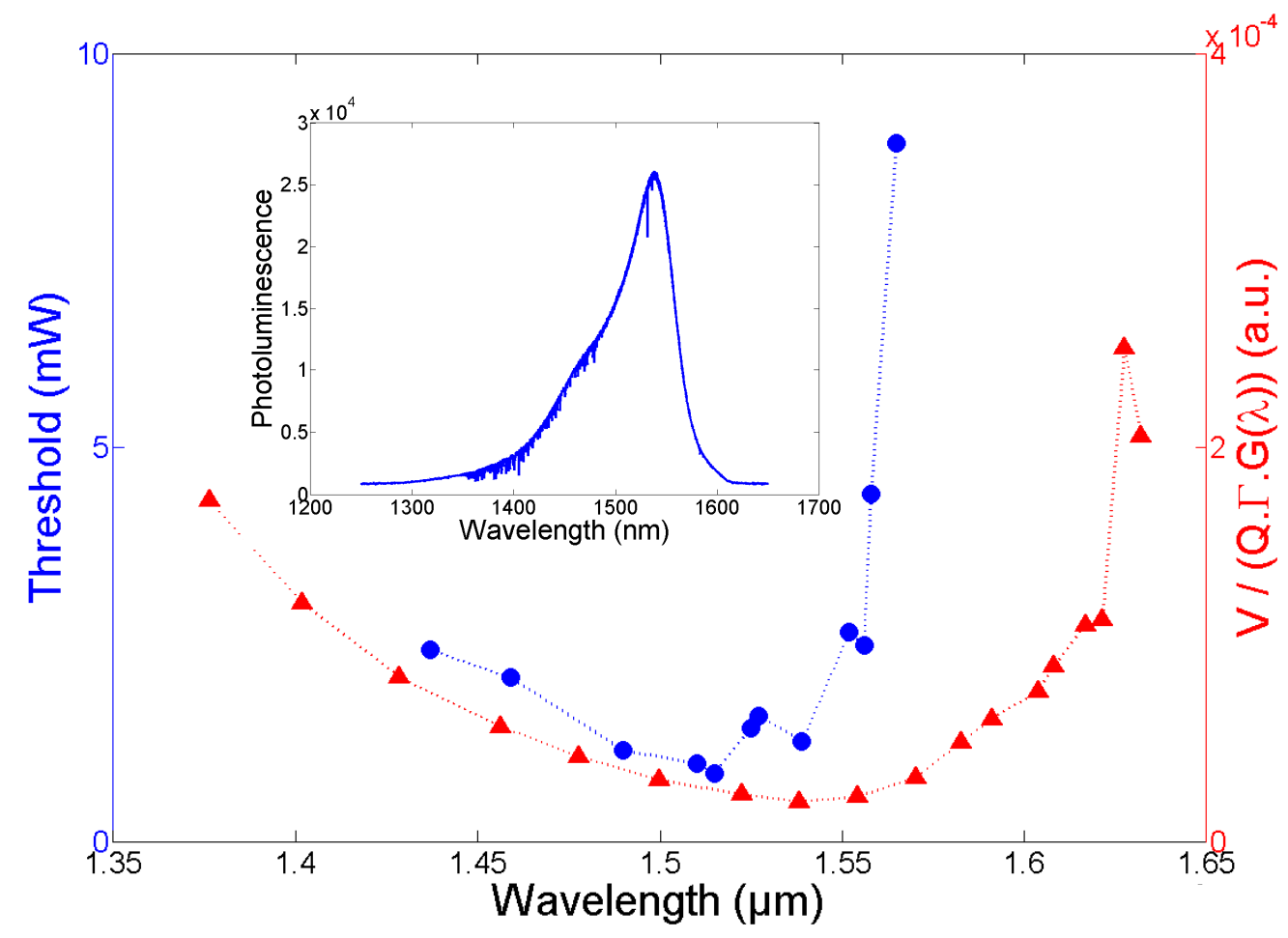


Figure 5

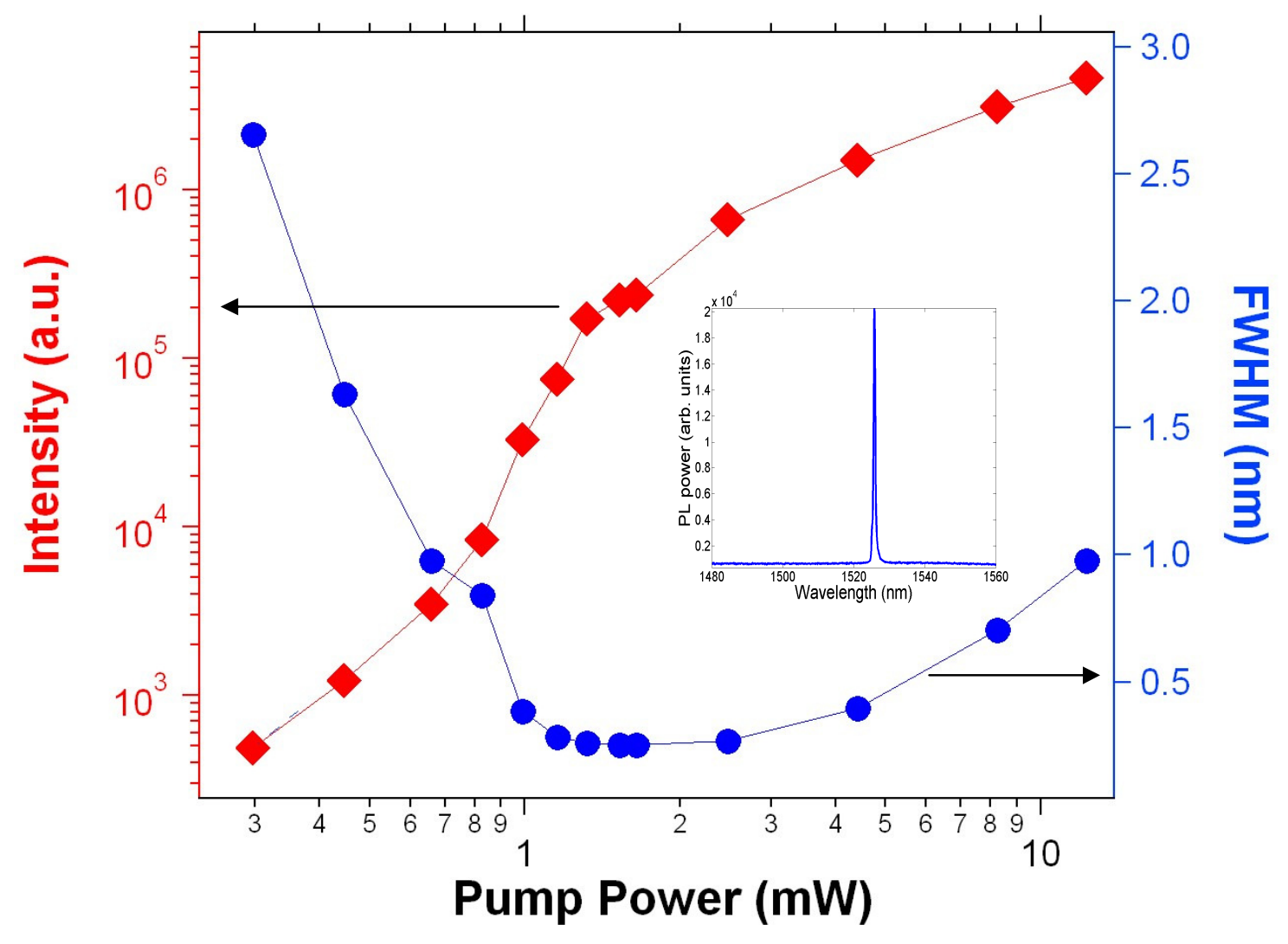

